\title{
Robust Mean Square Exponential Stability of Stochastic Interval Cellular Neural Networks with Time-delays
}

\author{
Zhichao Liu', Jinfang Han ${ }^{2}$ \\ ${ }^{1}$ College of Information Sci and Eng, Hebei University of Science and Technology, Shijiazhuang 050018, China \\ ${ }^{2}$ Inst of Eng Math, Hebei University of Science and Technology, Shijiazhuang 050018, China \\ jfhanemail@126.com, jfhan@hebust.edu.cn
}

\begin{abstract}
The problem of robust mean-square exponential stability for a class of stochastic interval cellular neural networks with time-delays is investigated. Firstly, a kind of equivalent description of this stochastic interval delayed cellular neural networks is presented. Then by using the Itô formula, Razumikhin theorems, Lyapunov function and norm inequalities, several simple sufficient conditions are obtained which guarantee the robust meansquare exponential stability of the stochastic interval cellular neural networks. and some recent results reported in the literatures are generalized. delays.

Index Terms - Robust Mean Square, Neural Networks, Time-
\end{abstract}

\section{I . Introduction}

In recent years, neural networks have been extensively investigated, and successfully applied in many areas such as combinatorial optimization, signal processing, pattern recognition and many other fields. However, all successful applications are greatly dependent on the dynamic behaviors of neural networks. As is well-known now, stability is one of the main properties of neural networks, which is a crucial feature in the design of neural networks. On the other hand, axonal signal transmission delays often occur in various neural networks, and may cause undesirable dynamic network behaviors such as oscillation and instability. Up to now, the stability analysis problem of neural networks with time-delay has been attracted a large amount of research interest and many sufficient conditions have been proposed to guarantee the asymptotic or exponential stability for the neural networks with various type of time delays such as constant, timevarying, or distributed. see for example [2], [4], [12-20] and [24], and the references therein.

Though the theoretical research on neural network has made great progress since it was born, but in many networks, such as in electronic neural networks, time delay can not be avoided. In fact, the stochastic perturbations can not be avoided either ${ }^{[6,7,10,11,12,21]}$. On the other hand, the system is unavoidable uncertainty, which is due to the existence of modeling errors, can also destroy the stability of the neural networks. So it is very important to discuss the stability and robustness of cellular network against such error and fluctuation $^{[8,12,14]}$. To overcome this difficulty, we will discuss the stability problem for a kind of stochastic interval cellular neural networks with time-delays (SICNND), and derive several exponential stability criteria for the cellular neural networks(SICNND).

This paper is organized as follows. In section 2, model description of the stochastic interval cellular neural networks with time-delays (SICNND), nomenclatures and lemma are given. In section 3, a kind of equivalent description of this stochastic interval delayed cellular neural networks and the idea for mean-square exponential stability are presented, and a set of some sufficient conditions is derived for the exponential stability of the stochastic interval cellular neural networks system. Finally, the conclusion is provided in section 4 .

\section{II . Problem Formulation and Preliminaries}

Consider an SICNND state equation as follows:

$d x(t)=\left[-D x(t)+A_{I} \sigma(x(t))+B_{I} \sigma\left(x\left(t-\tau_{1}\right)\right)\right] d t+f\left(t, x(t), x\left(t-\tau_{2}\right)\right) d w(t)$

where $x(t)=\left(x_{1}(t), x_{2}(t), \cdots x_{n}(t)\right)^{T} \in R^{n}$ is the state vector, and $D=\operatorname{diag}\left(d_{i i}\right)_{n \times n} \in R^{n \times n}, 0<d_{i i}, i=1,2, \cdots n$,

$$
\begin{aligned}
& A_{I}=\left\{A \mid A=\left(a_{i j}\right)_{n \times n}, p_{i j} \leq a_{i j} \leq q_{i j}, i, j=1,2, \cdots n\right\} \\
& B_{I}=\left\{B \mid B=\left(b_{i j}\right)_{n \times n}, r_{i j} \leq b_{i j} \leq f_{i j}, i, j=1,2, \cdots n\right\}
\end{aligned}
$$

are $n \times n$ interval matrices. Where $p_{i j}\left(r_{i j}\right)$ and $q_{i j}\left(f_{i j}\right)$ are exactly known, so that $P=\left(p_{i j}\right)_{n \times n}, Q=\left(q_{i j}\right)_{n \times n}, R=\left(r_{i j}\right)_{n \times n}, F=\left(f_{i j}\right)_{n \times n}$ are constant matrices. $\tau_{i} \in\left(R_{+},[0, \tau]\right), i=1,2, \tau>0$ are time lags. $\sigma(x)=\left(\sigma_{1}\left(x_{1}\right), \sigma_{2}\left(x_{2}\right), \cdots \sigma_{n}\left(x_{n}\right)\right)^{T}$, satisfies $\sigma_{i}\left(x_{i}\right)=\frac{1}{2}\left(\left|x_{i}+1\right|-\left|x_{i}-1\right|\right)(1 \leq i \leq n)$

$f\left(t, x(t), x\left(t-\tau_{2}\right)\right) d w(t) \quad$ denote random-perturbation. Where $w(t)=\left(w_{1}(t), w_{2}(t), \cdots w_{m}(t)\right)^{T}(m \leq n)$ is an m-dimensional Brownian motion which defined on a complete probability space $\left(\Omega, F, F_{t}, P\right)$ with a natural filtration $\left\{F_{t}\right\}_{t \geq 0}$, (i.e., $F_{t}==\sigma\{w(s): 0 \leq s \leq t\}$ ), and $\sigma(\cdot)$ is non-linear activity function, $f: R_{+} \times R^{n} \times R^{n} \rightarrow R^{n \times m}$ is an local Lipschitz continuous which satisfy linear growth condition[3]. that is, for any $t \geq 0$, there exists constants $c_{i}\left(d_{i}\right) \in[0, \infty)$ such that

$$
\left\{\begin{array}{l}
\left|\sigma_{i}\left(x_{i}\right)-\sigma_{i}\left(y_{i}\right)\right| \leq\left(1 \wedge c_{i}\right)\left|x_{i}-y_{i}\right|, \quad \forall x_{i} \neq y_{i} \\
|f(t, x, y)-f(t, \bar{x}, \bar{y})| \leq\left(1 \wedge d_{i}\right)(|x-\bar{x}|+|y-\bar{y}|)
\end{array}\right.
$$

where $(|x| \vee|\bar{x}| \vee|y| \vee|\bar{y}|) \leq 1$. And assume system (1) satisfy the initial condition $x(s)=\xi(s),-\tau \leq s \leq 0, \xi \in L_{F_{0}}^{2}\left([-\tau, 0], R^{n}\right)$, where $L_{F_{0}}^{2}\left([-\tau, 0], R^{n}\right)$ is a stochastic process $\xi(s)$ with $R^{n}$ value $(-\tau \leq s \leq 0)$, and $\xi(s)$ is $F_{0}-$ measurable, $\int_{-\tau}^{0} E|\xi(s)|^{2} d s<\infty$. We

know that the system (1) has a unique solution by the paper [3], and it is denoted by $x(t, \xi) \hat{=} x(t)$, it is also square integrable. 
In order to discuss the stability of system (1), assume, in the whole paper, $\forall t \in R_{+}: f(t, 0,0) \equiv 0$, and there exists $a_{1}, a_{2} \geq 0$, such that $\forall(t, x, y) \in R_{+} \times R^{n} \times R^{n}$, we have trace

$$
f^{T}(t, x, y) f(t, x, y) \leq a_{1}|x|^{2}+a_{2}|y|^{2}
$$

Particularly, $f^{T}\left(t, x(t), x\left(t-\tau_{i}\right)\right) f\left(t, x(t), x\left(t-\tau_{i}\right)\right) \leq a_{1}|x(t)|^{2}+a_{2}\left|x\left(t-\tau_{i}\right)\right|^{2}$ on the other hand, we can rewrite the interval matrices $A_{I}$ and $B_{I}$ in the following equivalent form:

$$
\begin{aligned}
& A_{I}=A_{0}+\Delta A \\
& B_{I}=B_{0}+\Delta B
\end{aligned}
$$

where

$$
\begin{aligned}
A_{0} & =\frac{1}{2}(P+Q) \\
B_{0} & =\frac{1}{2}(R+F) \\
\Delta A & =\sum_{i, j}^{n} \varepsilon_{i j} E_{i j}, E_{i j}=\frac{1}{2}\left(q_{i j}-p_{i j}\right) u_{i j}, u_{i j}=e_{i} e_{j}^{T} \\
\Delta B & =\sum_{t, k}^{n} \varepsilon_{t k} E_{t k}^{\prime}, E_{t k}^{\prime}=\frac{1}{2}\left(f_{t k}-r_{t k}\right) u_{t k}, u_{t k}=e_{t} e_{k}^{T}
\end{aligned}
$$

the uncertain parameters $\left|\varepsilon_{i j}\right| \leq 1,{ }_{i}$ and ${ }^{e_{j}}$ are unit vectors with only the ith and jth elements, being unity, respectively. $u_{i j}$ is a matrix with all entries being zero except its $(i, j)$ entry, which is unity. Also, we denote that (the case of $\varepsilon_{\mathrm{ij}}=1, \mathrm{i}, \mathrm{j}=1,2, \ldots \mathrm{n}$ ):

$$
\begin{aligned}
& E=\sum_{i, j=1}^{n} E_{i j}=\frac{1}{2}(Q-P) \\
& E^{\prime}=\sum_{t, k=1}^{n} E_{t k}^{\prime}=\frac{1}{2}(F-R)
\end{aligned}
$$

And it are called as maximum perturbation matrix of $A_{I}$ and $B_{I}$ respectively. Obviously, the $E \geq 0\left(E^{\prime} \geq 0\right)$. Using the matrices $A_{0}\left(B_{0}\right)$ and $E\left(E^{\prime}\right)$, the interval matrix $A_{I}$ and $B_{I}$ can be represented by

$$
\begin{aligned}
& A_{I}=[P, Q]=\left[A_{0}-E, A_{0}+E\right]=A_{0}+\Delta A,|\Delta A| \leq E \\
& B_{I}=[R, F]=\left[B_{0}-E^{\prime}, B_{0}+E^{\prime}\right]=B_{0}+\Delta B,|\Delta B| \leq E^{\prime}
\end{aligned}
$$

Where $\Delta A$ and $\Delta B$ are called as the bias matrices between $A_{I}, A_{0}$ and $B_{I}, B_{0}$, respectively. Also, we define

$$
M_{1}=\left(m_{i j}\right)_{n \times n}=Q-A_{0}, N_{1}=\left(n_{i j}\right)_{n \times n}=F-B_{0}
$$

Obviously, the $M_{1} \geq 0\left(N_{1} \geq 0\right)$, and we have

$$
|\Delta A| \leq\left|M_{1}\right|=M_{1} \leq E,|\Delta B| \leq\left|N_{1}\right|=N_{1} \leq E^{\prime}
$$

The following notations and engagements are used throughout this paper:

Given a square complex matrix $A=\left(a_{i j}\right) \in C^{n \times n}$,
$|A|=\left(\left|a_{i j}\right|\right)_{n \times n}$ denotes the modulus matrix of $A$, where $\left|a_{i j}\right|$ denotes the modulus (absolute value) of $a_{i j}$. For a real matrix $A=\left(a_{i j}\right) \in R^{n \times n}$ is nonnegative (positive) if its entries $a_{i j} \geq 0 \quad\left(a_{i j}>0\right)$ and $A \geq B \geq 0(A>B>0)$ means that the entriesof A and B satisfy $a_{i j} \geq b_{i j} \geq 0\left(a_{i j}>b_{i j}>0\right), i, j \in N=\{1,2, \cdots, n\}$, or $A-B \geq 0(A-B>0) \cdot \rho(A)$ the spectral radius of matrix $A$, and $I$ the identity matrix., $\lambda(A)$ denotes any eigenvalue of $A$. the operator norm or spectral norm of $A$ is denoted by $\|A\|$.

Lemma $1^{[22]}$ If $A$ and $B$ are quadratic matrices with nonnegative entries (some of the entries are positive) and $B \leq A$, then $\|B\|_{p} \leq\|A\|_{p}(\mathrm{p}=1,2, \infty)$.

Note that the equivalence of the norm, which can be written as $\|A\|_{p}=\|A\|(\mathrm{p}=1,2, \infty)$.

Associated (1), the following system is considered,

$d x(t)=\left[-D x(t)+A \sigma(x(t))+B \sigma\left(x\left(t-\tau_{1}\right)\right)\right] d t+f\left(t, x(t), x\left(t-\tau_{2}\right)\right) d w(t)$

$A \in A_{f}, B \in B_{I}$

\section{III . Main Results}

In view of the above section, a equivalent description of the systems (1) can be written as follows

$$
\begin{aligned}
d x(t)= & {\left[-D x(t)+\left(A_{0}+\Delta A\right) \sigma(x(t))+\left(B_{0}+\Delta B\right) \sigma\left(x\left(t-\tau_{1}\right)\right)\right] d t } \\
& +f\left(t, x(t), x\left(t-\tau_{2}\right)\right) d w(t) .
\end{aligned}
$$

$x(s)=\xi(s),-\tau \leq s \leq 0, \xi \in L_{F_{0}}^{2}\left([-\tau, 0], R^{n}\right)$

\section{Definition 2.1}

The stochastic interval cellular neural networks with time-delays (1) is said to be robust mean-square exponential stable, if for any $\forall A \in A_{I}, \forall B \in B_{I}$, the system (18) is mean-square exponential stable or (19) is mean-square exponential stable.

\section{Theorem 2.1}

If there exists positively definite matrix $G$ such that $\lambda_{\min }\left(G D+D^{T} G\right)>2\left(\left\|G A_{0}\right\|+\mid G M_{1} \|+\right.$

$+2\left(\left\|G B_{0}\right\|+\left\|G N_{1} D \sqrt{\lambda_{\max }(G) \lambda_{\min }^{-1}(G)}+\mid G\right\|\left(a_{1}+a_{2} \lambda_{\min }^{-1}(G) \lambda_{\max }(G)\right)\right.$

Then the trivial solution of systems (1) is robust meansquare exponential stable and almost surely exponential stable. Proof For $\forall\left(t, x, y_{1}, y_{2}\right) \in R_{+} \times R^{n} \times R^{n} \times R^{n}, \forall A \in A_{I}$, and $\forall B \in B_{I}$, (obviously $A=A_{0}+\Delta A, \quad B=B_{0}+\Delta B$ ), define the lyapunov function as follows: $V(x)=x^{T} G x$

In terms of Lemma $\mathbf{1}$ and Itô inequality, we have the following

$$
\begin{aligned}
L V(x(t))= & V_{x}(x)\left(-D x+A \sigma(x)+B \sigma\left(y_{1}\right)\right) \\
& +\frac{1}{2} t^{2} a c e f^{z}\left(t, x, y_{2}\right) V_{x z}(x) f\left(t, x, y_{2}\right)
\end{aligned}
$$




$$
\begin{aligned}
& =2 x^{T} G\left(-D x+A \sigma(x)+B \sigma\left(y_{1}\right)\right)+\operatorname{tracef}^{T}\left(t, x, y_{2}\right) G f\left(t, x, y_{2}\right) \\
& =2 x^{T} G\left(-D x+\left(A_{0}+\Delta A\right) \sigma(x)+\left(B_{0}+\Delta B\right) \sigma\left(y_{1}\right)\right) \\
& +\operatorname{tracef}^{T}\left(t, x, y_{2}\right) G f\left(t, x, y_{2}\right) \\
& \leq\left(-\lambda_{\min }\left(G D+D^{T} G\right)+2\left\|G\left(A_{0}+\Delta A\right)\right\|+\left\|G\left(B_{0}+\Delta B\right)\right\| \beta+\|G\| a_{1}\right)|x|^{2} \\
& +\left\|G\left(B_{0}+\Delta B\right)\right\| \beta^{-1}\left|y_{1}\right|^{2}+\|G\| a_{2}\left|y_{2}\right|^{2} \\
& \leq\left(-\lambda_{\min }\left(G D+D^{T} G\right)+2\left(\left\|G A_{0}\right\|+\left\|G M_{1}\right\|+\left(\left\|\left.G B_{0}\left|+\left\|G V_{1}|D \beta+\| G| a_{1}\right)\right| x\right|^{2}\right.\right.\right.\right. \\
& +\left(\left\|G B_{0}\right\|+\left\|\left.G v_{1}\left|D \beta^{-1}\right| y_{1}\right|^{2}+\right\| G \| a_{2}\left|y_{2}\right|^{2}\right. \\
& \leq-\lambda_{1} V(x)+\lambda_{2} V\left(y_{1}\right)+\lambda_{3} V\left(y_{2}\right) \\
& \text { Where } \quad \lambda_{1}=\left(\lambda_{\min }\left(G D+D^{T} G\right)-2\left(\left\|G A_{0}\right\|+\left\|G M_{1}\right\|\right)-\right. \\
& \left.\quad-\left(\left\|G B_{0}\right\|+\left\|G N_{1}\right\|\right) \beta+\|G\| a_{1}\right) \lambda_{\max }^{-1}(G) \\
& \quad \lambda_{2}=\left(\left\|G B_{0}\right\|+\left\|G N_{1}\right\|\right) \beta^{-1} \lambda_{\min }^{-1}(G), \\
& \quad \lambda_{3}=\|G\| a_{2} \lambda_{\min }^{-1}(G), \beta=\sqrt{\lambda_{\min }^{-1}(G) \lambda_{\max }(G)}
\end{aligned}
$$

By the condition (20), it is easy to show that, $\lambda_{1}>\lambda_{2}+\lambda_{3}$. Thus, by virtue of Razumikhin-type theorem in [3,5], we know the trivial solution of system (18) is mean-square exponential stable and almost surely exponential stable. Thus the theorem 2.1 is proved by the arbitrariness of $A, B$ and Definition 2.1.

\section{Theorem 2.2}

If there exists positively definite matrix $G$ such that

$$
\lambda_{\min }\left(G D+D^{T} G\right)>2\left(\left\|G\left(A_{0}+B_{0}\right)\right\|+\| G\left(M_{1}+N_{1} \|\right)+2\left(\left\|G B_{0}\right\|\right.\right.
$$$$
\left\|G N_{i} D \sqrt{H \lambda_{\max }(G) \lambda_{\min }^{-1}(G)}+\right\| G \|\left(a_{1}+a_{2} \lambda_{\min }^{-1}(G) \lambda_{\max }(G)\right)
$$

Then the trivial solution of systems (1) is robust mean-square exponential stable and almost surely exponential stable. Where

$$
H=\left[\tau\left(\|D\|+\left\|A_{0}\right\|+\left\|B_{0}\right\|\right)+\sqrt{\tau\left(\alpha_{1}+\alpha_{2}\right)}\right]^{2}
$$

Proof Let $x(t)$ be a solution of systems(1), then $\forall i=1,2$, by the Hölder inequality, we have

$$
\begin{aligned}
& E\left|x(t)-x\left(t-\tau_{i}\right)\right|^{2} \leq\left(\|D\| \tau+\left\|A_{0}+\Delta A\right\| \tau+\left\|B_{0}+\Delta B\right\| \tau\right. \\
& \left.+\sqrt{z\left(\alpha_{1}+\alpha_{2}\right)}\right)\left(\frac{1}{|F| z} E\left|\int_{t-z_{t}}^{t}-D x(s) d\right|^{2}\right. \\
& +\frac{1}{\left|A_{0}+\Delta A\right|:} E\left|\int_{t-\tau_{1}}^{t}\left(A_{0}+\Delta A\right) \sigma(x(s)) d s\right|^{2} \\
& +\frac{1}{\left|B_{0}+\Delta E\right|=} E\left|\int_{t-\tau_{i}}^{t}\left(B_{0}+\Delta B\right) \sigma\left(x\left(s-\tau_{i}\right)\right) d s\right| \\
& \left.\left.+\frac{1}{\sqrt{\left(a_{4}+\infty_{2}\right)}} E \mid \int_{t-\varepsilon_{1}}^{t} f d w(s)\right)\left.d s\right|^{2}\right),
\end{aligned}
$$

$$
\begin{aligned}
& +\frac{1}{\left\|A_{0}+\Delta A\right\| \tau} E\left|\int_{t-\tau_{i}}^{t}\left(A_{0}+\Delta A\right) \sigma(x(s)) d s\right|^{2}+ \\
& +\frac{1}{\left\|B_{0}+\Delta B\right\| \tau} E\left|\int_{t-\tau_{i}}^{t}\left(B_{0}+\Delta B\right) \sigma\left(x\left(s-\tau_{i}\right)\right) d s\right|^{2} \\
& \left.\left.+\frac{1}{\sqrt{\tau\left(\alpha_{1}+\alpha_{2}\right)}} E \mid \int_{t-\tau_{i}}^{t} f d w(s)\right)\left.d s\right|^{2}\right),
\end{aligned}
$$

By employing the Hölder inequality go on, we can get

$$
\begin{aligned}
& E\left|x(t)-x\left(t-\tau_{i}\right)\right|^{2} \leq\left(\|D\| \tau+\left\|A_{0}+\Delta A\right\| \tau\right. \\
& \left.\left\|B_{0}+\Delta B\right\| \tau+\sqrt{\tau\left(\alpha_{1}+\alpha_{2}\right)}\right)\left(\frac{1}{|\boldsymbol{T}|^{2}}\|D\|^{2} \tau^{2} \sup _{-2 \operatorname{sits} 0} E|x(t+\theta)|^{2}\right. \\
& +\frac{1}{\left|A_{0}+\Delta A\right| \mid}\left\|A_{0}+\Delta A\right\|^{2} \tau^{2} \sup _{-2 \mathrm{r} \forall \operatorname{sis}_{0}} E \mid\left(\left.x(t+\theta)\right|^{2}\right. \\
& +\frac{1}{\left|B_{0}+\Delta B\right|^{2}}\left\|B_{0}+\Delta B\right\|^{2} \tau^{2} \sup _{-2 \pi \operatorname{ses} 0} E \mid\left(\left.x(t+\theta)\right|^{2}\right. \\
& \left.\left.+\frac{1}{\sqrt{z\left(\alpha_{1}+\alpha_{2}\right)}} E \mid \int_{t-\tau_{1}}^{t} f d w(s)\right)\left.d s\right|^{2}\right),
\end{aligned}
$$

By virtue of lemma2.3 in [7] and note that (5), we have

$$
\begin{aligned}
& E\left|x(t)-x\left(t-\tau_{i}\right)\right|^{2} \leq\left(\|D\| \tau+\left\|A_{0}+\Delta A\right\| \tau\right. \\
& \left.+\left\|B_{0}+\Delta B\right\| \tau+\sqrt{\tau\left(\alpha_{1}+\alpha_{2}\right)}\right)\|D\| \tau \sup _{-2 \tau \leq \theta \leq 0} E|x(t+\theta)|^{2} \\
& +\left\|A_{0}+\Delta A\right\| \tau \sup _{-2 \tau \leq \theta \leq 0} E \mid\left(\left.x(t+\theta)\right|^{2}+\right. \\
& +\left\|B_{0}+\Delta B\right\| \tau \sup _{-2 \tau \leq \theta \leq 0} E \mid\left(\left.x(t+\theta)\right|^{2}\right. \\
& +\frac{1}{\sqrt{\tau\left(\alpha_{1}+\alpha_{2}\right)}} \tau\left(\alpha_{1}+\alpha_{2}\right) \sup _{-2 \tau \leq \theta \leq 0} E \mid\left(\left.x(t+\theta)\right|^{2}\right. \\
& \leq\left(\|D\| \tau+\left\|A_{0}+\Delta A\right\| \tau+\left\|B_{0}+\Delta B\right\| \tau+\sqrt{\tau\left(\alpha_{1}+\alpha_{2}\right)}\right) \\
& +\left(\|D\| \tau \sup _{-2 \tau \leq \theta \leq 0} E|x(t+\theta)|^{2}+\left\|A_{0}+\Delta A\right\| \tau \sup _{-2 \tau \leq \theta \leq 0} E \mid\left(\left.x(t+\theta)\right|^{2}\right.\right. \\
& +\left\|B_{0}+\Delta B\right\| \tau \sup _{-2 \tau \leq \theta \leq 0} E \mid\left(\left.x(t+\theta)\right|^{2}\right. \\
& +\sqrt{\tau\left(\alpha_{1}+\alpha_{2}\right)} \sup _{-2 \tau \leq \theta \leq 0} E \mid\left(\left.x(t+\theta)\right|^{2}\right) \\
& \leq\left(\|D\| \tau+\left\|A_{0}+\Delta A\right\| \tau+\left\|B_{0}+\Delta B\right\| \tau\right. \\
& \left.+\sqrt{\tau\left(\alpha_{1}+\alpha_{2}\right)}\right)\left(\|D\| \tau+\left\|A_{0}+\Delta A\right\| \tau\right. \\
& \left.+\left\|B_{0}+\Delta B\right\| \tau+\sqrt{\tau\left(\alpha_{1}+\alpha_{2}\right)}\right)\left(\sup _{-\tau \leq 0 \leq 0} E \mid\left(\left.x(t+\theta)\right|^{2}\right)\right. \\
& \leq\left(\|D\| \tau+\left\|A_{0}+\Delta A\right\| \tau+\left\|B_{0}+\Delta B\right\| \tau+\right. \\
& \left.+\sqrt{\tau\left(\alpha_{1}+\alpha_{2}\right)}\right)\left(\|D\| \tau+\left\|A_{0}+\Delta A\right\| \tau+\left\|B_{0}+\Delta B\right\| \tau+\right. \\
& \left.+\sqrt{\tau\left(\alpha_{1}+\alpha_{2}\right)}\right)\left(\sup _{-\tau \leq \theta \leq 0} E \mid\left(\left.x(t+\theta)\right|^{2}\right)\right.
\end{aligned}
$$




$$
\begin{aligned}
& =\left[\|D\| \tau+\left\|A_{0}+\Delta A\right\| \tau+\left\|B_{0}+\Delta B\right\| \tau+\sqrt{\tau\left(\alpha_{1}+\alpha_{2}\right)}\right]^{2} . \\
& \cdot\left(\sup _{-2 \tau \leq \theta \leq 0} E \mid\left(\left.x(t+\theta)\right|^{2}\right)\right. \\
& \leq\left[\left(\|D\|+\left\|A_{0}\right\|+\left\|B_{0}\right\|+\left\|M_{1}\right\|+\left\|N_{1}\right\|\right) \tau+\sqrt{\tau\left(\alpha_{1}+\alpha_{2}\right)}\right]^{2} . \\
& \cdot\left(\sup _{-2 \tau \leq \theta \leq 0} E \mid\left(\left.x(t+\theta)\right|^{2}\right),\right. \\
& i . e ., E\left|x(t)-x\left(t-\tau_{i}\right)\right|^{2} \leq \\
& \leq\left[\left(\|D\|+\left\|A_{0}\right\|+\left\|B_{0}\right\|+\left\|M_{1}\right\|+\left\|N_{1}\right\|\right) \tau+\sqrt{\tau\left(\alpha_{1}+\alpha_{2}\right)}\right]^{2} . \\
& \cdot\left(\sup _{-2 \tau \leq \theta \leq 0} E \mid\left(\left.x(t+\theta)\right|^{2}\right)\right. \\
& \hat{=} H\left(\sup _{-2 \tau \leq \theta \leq 0} E \mid\left(\left.x(t+\theta)\right|^{2}\right), \cdots \cdots \cdots \cdots \cdots(25)\right.
\end{aligned}
$$

Now, For $\forall A \in A_{I}, \forall B \in B_{I}$ ( obviously $A=A_{0}+\Delta A$, $\left.B=B_{0}+\Delta B\right)$, define lyapunov function as follows: $V(x)=x^{T} G x$ by using the Itô inequality, and notice that (5), we have

$$
\begin{aligned}
& L V(x(t))=2 x^{T}(t) G(-D x(t))+(A+B) \sigma(x(t))+ \\
& +2 x^{T}(t) G B\left(\sigma\left(x\left(t-\tau_{i}\right)-\sigma(x(t))\right)+\text { tracef }^{T} G f\right. \\
& =2 x^{T}(t) G(-D x(t))+\left(A_{0}+\Delta A+B_{0}+\Delta B\right) \sigma(x(t)) \\
& +2 x^{T}(t) G\left(B_{0}+\Delta B\right)\left(\sigma\left(x\left(t-\tau_{i}\right)\right)-\sigma(x(t))\right)+\text { tracef }^{T} G f \\
& \leq-\left[\lambda_{\min }\left(G D+D^{T} G\right)-2\left\|G\left(A_{0}+B_{0}\right)\right\|-\right. \\
& \left.-\left\|G B_{0}\right\| \sqrt{H \lambda_{\max }(G) \lambda_{\min }^{-1}(G)}-\|G\| \alpha_{1}\right]|x(t)|^{2} \\
& +\frac{\left\|G B_{0}\right\|}{\sqrt{H \lambda_{\max }(G) \lambda_{\min }^{-1}(G)}}\left|x\left(t-\tau_{1}\right)-x(t)\right|^{2}+\|G\| \alpha_{2}\left|x\left(t-\tau_{2}\right)\right|^{2}
\end{aligned}
$$

by the (22) we have

$$
\begin{aligned}
& \lambda_{\text {min }}\left(G D+D^{T} G\right)>2\left\|G\left(A_{0}+B_{0}\right)\right\|+ \\
& +2\left\|G B_{0}\right\| \sqrt{H \lambda_{\text {max }}(G) \lambda_{\text {min }}^{-1}(G)}+\|G\|\left(a_{1}+a_{2} \lambda_{\text {min }}^{-1}(G) \lambda_{\text {max }}(G)\right),
\end{aligned}
$$

Thus, by virtue of (25),(27), from the (26) we can get

$$
\begin{aligned}
& E L V(x(t)) \leq-\left[\lambda_{\min }\left(G D+D^{z} G\right)-2\left\|G\left(A_{0}+B_{0}\right)\right\|\right. \\
& \left.\_\left\|G B_{0}\right\| \sqrt{H \lambda_{\max }(G) \lambda_{\min }^{-1}(G)}-\|G\| \alpha_{1}\right] \lambda_{\operatorname{mix}}^{-1}(G) E V(x(t))
\end{aligned}
$$

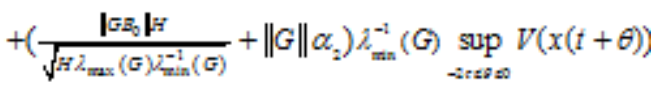

From the (27), it is easy to see that there is exist $q>1$ such that

$$
\begin{aligned}
& -\lambda=-1 \lambda_{\min }\left(G D+D^{T} G\right)-2 \mid G\left(A_{0}+B_{0}\right)\|-\| G B_{0} \| \sqrt{H \lambda_{\operatorname{mx}}(G) \lambda_{\min }^{-1}(G)}
\end{aligned}
$$

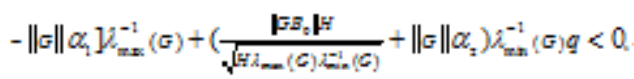

thus, as the inequality $E V(x(t+\theta))<q E V(x(t)),-2 \tau \leq \theta \leq 0$ holds, by the (28),(29), we can obtain

$$
\operatorname{ELV}(x(t)) \leq-\lambda E V(x(t))
$$

Thus, by virtue of Razumikhin-type theorem in [3,5], we know that the trivial solution of system (18) is robust mean-square exponential stable and almost surely exponential stable. Thus the Theorem 2.2 is proved by the arbitrariness of $A, B$ and Definition 2.1.

\section{Conclusions}

The above we have discussed the robust mean-square exponential stability problem for a class of stochastic interval cellular neural networks with time-delays. by means of the Itô formula, Razumikhin theorems, Lyapunov function and norm inequalities, several sufficient conditions are obtained which guarantee the mean-square exponential stability of the stochastic interval cellular neural networks with time-delays. and some recent results reported in the literatures are generalized.

\section{V . Acknowledgment}

This paper is supported by the National Natural Science Foundation of China (70271006, 60674107), and by the Natural Science Foundation of Hebei Province (F2006000571 , F2006000343).

\section{References}

[1] Liao X F., "Novel robust stability criteria for interval-delayed Hopfield neural networks". IEEE Trans. Circuits and Systems, vol.48, no.11, pp. $1355-1359.2001$.

[2] Liao X F and Yu J., "Robust stability for interval Hopfield neural networks with time delays".IEEE Trans. Neural Networks, No.9, pp. 1042-1046, 1998,.

[3] Mao X.R.., "Exponential Stability of stochastic differential Equationssystems". New York: Marcel Dekker. 1994

[4] Mao X.R., Selfridge C., "Stability of stochastic systems with time delays". Systems \& Control Letters, vol.42, no.2, pp. 279-290. 2001.

[5] Mao X.R., "Razumikhin-type theorems on exponential stability of stochastic functional differential equations". Stochastic Processes and their Applications, vol.65, no.2, pp.233-250. 1996.

[6] Shen Yi, Jiang Minghui and YaoHongshan, "Exponential Stabiluty of Cellular Neural Networks". Acta Mathematica Scientia (in Chinesee ), vol.25, no.2, pp.264-268. 2005.

[7] Mao X. R., Shah A., "Exponential stability of stochastic differential delay equations". Stochastics and stochastics reports, vol.60,no.2, pp. 135-153. 1997

[8] Liao X. X.,"Novel robust stability criteria for interval-delayed Hopfield neural networks". IEEE trans on circuits and systems, vol.48, no.11,pp. 1355-1359. 2001.

[9] Shen Yi, Liao Xiao-xin. "Exponential Stabiluty of Hopfield-Type Stochastic Delay Neural Networks", Acta Mathematica Scientia(in Chinese),vol.19, no2, pp.211-218, 1999.

[10] Liao X. X, Mao X. R.,"Exponential stability and instability of stochastic neural networks". Stochast. Anal. Appl., vol. 4, no2, pp.165-168. 1996.

[11] Liao X. X, Mao X. R., "Stability of stochastic neural networks". Neural.,Parallel Science Computing, vol.4, no.2, pp.205-224, 1996.

[12] Mao X. R., Selfridge C.,"Stability of stochastic systems with time delays". Systems \& Control Letters,vol.42, no.2, pp. 279-290, 2001.

[13] Jinde Cao, Qing Li."On the exponential stability and periodic solutions of delayed cellular neural networks". Journal of mathematical analysis and applications,vol.25,no.2,pp.50-64,2000 . 
[14] Liao Xiao-xin, Xie Dong-mei. "Global exponential stability of hopfield neural networks with time-varying delays," Acta electronica sinica(in Chinese),vol.28, no4, pp.87-90, 2000.

[15] Wang Linshan, Xu Dao-yi. "Stability analysis of hopfield neural networks with time delay," Applied mathematics and mechanics(in Chinese),vol.23, no.1, pp. 59-64,2002.

[16] Sabri Arik., "lobal asymptotic stability analysis of bi-directional associative memory neural networks with time delays". IEEE Trans. Neural Netw., Vol.16, No.3, pp. 580-586,2005.

[17] M. Forti, S. Manetti and M. Marini, "Necessary and sufficient condition for absolute stability of neural networks", IEEE Trans Circuits Syst I 41, pp. 491-494. 1994.

[18] S. Arik, "Stability analysis of delayed neural networks", IEEE Trans Circuits Syst I 47, pp. 1089-1092, 2000.

[19] J. Cao and J. Wang, Global asymptotic and robust stability of recurrent neural networks with time delays, IEEE Trans Circuits Syst I 52, pp. 417-426, 2005.
[20] Y. Zhang, P.A. Heng and K.S. Leung, Convergence analysis of cellular neural networks with unbounded delays, IEEE Trans Circuits Syst I 48, pp. 680-687, 2001.

[21] T.P. Chen and S. Amari, Stability of asymmetric Hopfield networks, IEEE Trans Neural Networks 12, pp. 159-163, 2001.

[22] Z.Bubnicki. "General approach to stability and stabilization for a class of uncertain discrete non-linear systems". Int.J. Control, Vol.73, No.14, pp. 1298-1306, 2000.

[23] Han Jin-fang, Li Yong-wei. "New criteria of global exponential stability for cellular neural networks," J. Hebei Univ. of Sci. \& Tech., vol.22, no.3, pp 37-40, 2001.

[24] Zhang J,“Globally exponential stability of neural networks with variable delays”. IEEE Trans Circuits Systems, Vol.50, No.2,pp.288-291, 2003.

[25] X. Liao, G.Chen, and E Sanchez. "Delay - dependent exponential stability analysis of delayed neural networks: an LMI Approach", Neural Networks, Vol.15, pp.855-866,2002.

[26] Jinde Cao. "Global stability conditions for delayed CNNs," IEEE Trans. Circuits Syst. I, Vol.48,No2.pp. 1330-1333, 2001. 